Article

\title{
Parameters identification for inverse option problems using Markov Chain Monte Carlo methods
}

\author{
Yasushi Ota ${ }^{1, *(\mathbb{D})}$ and Yu Jiang ${ }^{2}$ \\ 1 Department of Management, Okayama University of Science, 1-1 Ridaicyou, Okayama City, Okayama \\ 7000005 Japan; yota@mgt.ous.ac.jp \\ 2 School of Mathematics, Shanghai University of Finance and Economics, 777 Guoding Rd.,Shanghai,200433,P. \\ R. China; jiang.yu@mail.shufe.edu.cn \\ * Correspondence: yota@mgt.ous.ac.jp; Tel.: +81-086-256-9805
}

\begin{abstract}
This paper investigates the inverse option problems (IOP) in the extended Black-Scholes model arising in financial market. We identify the volatility and the drift coefficient from the measured data in financial markets using a Bayesian inference approach, which is presented as an IOP solution. The posterior probability density function of the parameters is computed from the measured data. The statistics of the unknown parameters are estimated by a Markov Chain Monte Carlo (MCMC) algorithm, which exploits the posterior state space. The efficient sampling strategy of the MCMC algorithm enables us to solve inverse problems by the Bayesian inference technique. Our numerical results indicate that the Bayesian inference approach can simultaneously estimate the unknown trend and volatility coefficients from the measured data.
\end{abstract}

Keywords: Inverse problem; Option pricing; Bayesian inference approach

\section{Introduction}

The technique of inverse problems for a partial differential equation of a parabolic type is developed and used in various fields, such as Inverse heat transfer problems(IHTP), Inverse heat conduction problems(IHCP), Inverse option problems(IOP), etc[1,2,4].

In this paper we consider the backward parabolic eauation:

$$
\left\{\begin{array}{cc}
\frac{\partial u}{\partial t}+\frac{1}{2} \sigma(x, t)^{2} x^{2} \frac{\partial^{2} u}{\partial x^{2}}+\mu(x, t) x \frac{\partial u}{\partial x}-r u=0, & (x, t) \in(0, \infty) \times[0, T), \\
\left.u(x, t)\right|_{t=T}=\Phi(x, T), & x \in(0, \infty) .
\end{array}\right.
$$

where $u(x, t)$ is the price for a derivative, such as an option, bond, interest rate, futures, foreign exchange, etc. Moreover, $x$ in the underlying asset price, $t$ is the time, $\sigma(x, t)$ and $\mu(x, t)$ are the drift and volatility coefficient of the process $x$, the interest rate $r$ is a nonnegative constant, and $K$ is the strike price and $T$ is the maturity of the underlying asset, and $\Phi(x, T)$ is a suitable initial condition. Now, we are interested in the following inverse option problem(IOP): Let the current time $t^{*}$ be given , and determine simultaneously $\mu(x, t)$ and $\sigma(x, t)$ from the observation of data $u\left(x, t^{*}\right), x \in \omega$, where $\omega$ is the interval.

IOP in mathematical finance were started by Dupire [8]. He derived the option premium $U(T, K)$ as a solution $u(\cdot, \cdot ; T, K)$ to the dual equation of Black-Schoels equation, which is $\mu=r$ in (1), with respect to the strike price $K$ and maturity $\mathrm{T}$ as follows:

$$
\frac{\partial U}{\partial T}-\frac{1}{2} \sigma(T, K)^{2} K^{2} \frac{\partial^{2} U}{\partial K^{2}}+r K \frac{\partial U}{\partial K}=0 .
$$


If the option price and its derivative can be determined for all possible $T$ and $K$, then the local volatility function $\sigma(T, K)$ can be directly derived from Eq.(2) as

$$
\sigma(T, K)^{2}=\frac{\frac{\partial U}{\partial T}+r K \frac{\partial U}{\partial K}}{\frac{1}{2} K^{2} \frac{\partial^{2} U}{\partial K^{2}}} .
$$

Using this approach, we can deduce the local volatility function from the quoted option prices in the financial market. Bouchouev and Isakov [4], Bouchouev et al. [5], and Ota and Kaji [24], by using a linearization method, considered the following form of the time-independent local volatility function $\sigma^{2}(K)$ :

$$
\frac{1}{2} \sigma^{2}(K)=\frac{1}{2} \sigma_{0}^{2}+f(K)
$$

where $f$ is a small perturbation of the constant volatility $\sigma_{0}$. Moreover, Mitsuhiro and Ota [23], Korolev et al. [17] and Doi and Ota [9] used the extended Black-Scholes equation (1) and then reconstructed the trend function by linearization method. The above studies provided point estimates of unknown parameters by exact determination or least squares optimization, without rigorously examining and considering the measurement errors in the inverse solutions. In [25] we reconstruct the parameters not by linearizing the inverse problems but by applying Bayesian inference to IOP.

In this paper, we investigate the Binary Option Problem, which has an initial condition $\Phi(x, T)=$ $H(x-K)$ in (1), where $H$ is the Heviside function, that is,

$$
H(x-K)= \begin{cases}1 & x \geq K \\ 0 & x<K .\end{cases}
$$

And we attempt a parameter reconstruction by a statistical method that simultaneously estimates the unknown trend and volatility coefficients from the measured data.

Bayesian inference approach solves an inverse problem by formulating a complete probabilistic description of the unknowns and uncertainties from the given measured data (see [16]). Incorporating the likelihood function with a prior distribution, the Bayesian inference method provides the posterior probability density function (PPDF). Owing to the recent developments in Bayesian inference work, including Bayesian inference approach by efficient sampling methods such as Markov Chain Monte Carlo (MCMC), we can apply the Bayesian inference technique to inverse problems in remote sensing [11], seismic inversion [21], heat conduction problems [29], [30] and various other real-world problems. Moreover, several prior publications such as $[6,14,15,27,28]$ are related to option pricing based on Bayesian inference. In those publications, the option prices are usually computed by using the analytical solution (or so-called Black-Scholes formula) or applying of Monte Carlo simulation of original stochastic differential equation under an assumption which the volatility is constant.

This paper is divided into five parts. Our inverse problem is mathematically formulated in Section 2. Section 3 outlines the general Bayesian framework for solving inverse problems and discusses the numerical exploration of the posterior state space by the MCMC method. In Section 4, we discretize our inverse problem and reconstruct the parameters by a numerical algorithm. We then discuss various aspects of our results through numerical examples. Concluding remarks are given in Section 5. 


\section{2. Mathematical formulation of IOP}

In this paper, we consider that the volatility is a constant $\left(\sigma(x, t) \equiv \sigma_{0}\right)$ and the initial condition is a step function in (1):

$$
\left\{\begin{array}{cl}
\frac{\partial u}{\partial t}+\frac{1}{2} \sigma_{0}^{2} x^{2} \frac{\partial^{2} u}{\partial x^{2}}+\mu(x, t) x \frac{\partial u}{\partial x}-r u=0 & (x, t) \in(0, \infty) \times[0, T), \\
\left.u(x, t)\right|_{t=T}=H(x-K) & x \in(0, \infty) .
\end{array}\right.
$$

47 First, we check an idea of Dupire[8] and derive the partial differential equation dual to (4).

We set

$$
G(x, t ; K, T)=-\frac{\partial u(x, t ; K, T)}{\partial K}
$$

and then $G(S, t ; K, T)$ satisfies the differential equation (4), and

$$
G(x, T ; K, T)=\delta(x-K) .
$$

According to Friedman[10], $G(x, t ; K, T)$ satisfies for fixed $(x, t)$ as a function of $(K, T)$ the following differential equation and initial condition:

$$
\left\{\begin{array}{c}
\frac{\partial G}{\partial T}-\frac{1}{2} \frac{\partial^{2}}{\partial K^{2}}\left(\sigma_{0}^{2} K^{2} G\right)+\frac{\partial}{\partial K}(\mu(K, T) K G)+r G=0(x, t) \in(0, \infty) \times[0, T), \\
\left.G(x, t ; K, t)\right|_{t=T}=\delta(x-K) \quad x \in(0, \infty) .
\end{array}\right.
$$

48 Then, we use the definition of $G(S, T ; K, T)$, and integrate the equation (7) from $K$ to $\infty$. The third term

49 in the left-hand side can be integrated by parts as follows

$$
\int_{K}^{\infty} \frac{\partial}{\partial \xi}(\mu(\xi, T) \xi G) d \xi=\mu(K, T) K \frac{\partial u}{\partial K}
$$

where we have used the following behaviour at infinity

$$
u, K \frac{\partial u}{\partial K}, K^{2} \frac{\partial^{2} u}{\partial K^{2}} \rightarrow 0 \quad \text { as } K \rightarrow \infty
$$

Consequentry, we can obtain the following dual equation for $u(\cdot ; K, T)$

$$
\frac{\partial u}{\partial T}-\frac{1}{2} \sigma_{0} K^{2} \frac{\partial^{2} u}{\partial K^{2}}-\left(\sigma_{0}-\mu(K, T)\right) K \frac{\partial u}{\partial K}+r u=0 .
$$

Now, the substitution

$$
\begin{gathered}
y=\log \frac{K}{x}, \quad \tau=T-t, \\
\mu(y)=\mu(K, T), \quad U(y, \tau)=u(x, t ; K, T)
\end{gathered}
$$

transforms the equation and the initial condition (4) into

$$
\left\{\begin{array}{c}
\frac{\partial U}{\partial \tau}-\frac{\sigma_{0}^{2}}{2} \frac{\partial^{2} U}{\partial y^{2}}-\left(\frac{\sigma_{0}^{2}}{2}-\mu(y)\right) \frac{\partial U}{\partial y}+r U=0 \quad(y, \tau) \in \mathbf{R} \times\left(0, \tau^{*}\right), \\
U(y, 0)=H(-y) \quad y \in \mathbf{R},
\end{array}\right.
$$

where $\tau^{*}=T-t^{*}$ and $t^{*}$ is the current time. 


\section{Bayesian inference approach to IOP}

The Bayesian inference approach is now widely used with great successes for solving a variety of inverse problem (see for example [16]). The solution of the Bayesian inference approach is estimated not as single-valued, but as the posterior conditional mean (CM)

$$
\theta_{\mathbf{C M}}:=\int \theta f(\theta \mid Y) \mathrm{d} \theta,
$$

of the unknown parameters $\theta$ given the measured data $Y$. Here, according to the Bayes' theorem, the posterior probability density function (PPDF) is defined as follows:

$$
f(\theta \mid Y)=\frac{f(Y \mid \theta) f(\theta)}{f(Y)} .
$$

i.e. the posterior probability of a hypothesis is proportional to the product of its likelihood and its prior probability. The likelihood function $f(Y \mid \theta)$ is then given as

$$
f(Y \mid \theta)=\exp \left\{-\frac{(Y-F(\theta))^{T}(Y-F(\theta))}{2 \Sigma_{\varepsilon}^{2}}\right\} .
$$

It is hard to know the explicit form of $f(\theta \mid Y)$ in (11), Markov chain Monte Carlo (MCMC) algorithm given in Robert and Casella [26] can be applied to obtain a set of samples $\theta_{k}(k=1, \cdots, K)$ and these independent samples can approach the distribution $f(\theta \mid Y)$. Also the posterior conditional mean comes to

$$
\theta_{\mathbf{C M}} \approx \frac{1}{K} \sum_{k=1}^{K} \theta_{k} .
$$

This is the solution of our IOP under the meaning of statistics. 
In this paper, we employs a typical MCMC algorithm called the Metropolis-Hastings (M-H) algorithm (see Metropolis et al. [22]; Hastings [12]). M-H Algorithm given below builds its Markov chain by accepting or rejecting samples extracted from a proposed distribution. $\mathbf{M}-\mathbf{H}$ Algorithm is generally used in Bayesian inference approach (cf. [16]).

\section{M-H Algorithm}

- Step1: Generate $\theta^{\prime} \sim q\left(\cdot \mid \theta_{k}\right)=N\left(\theta_{k}, \gamma^{2}\right)$ (the normal distribution) with a given stander derivation $\gamma>0$ for given $\theta_{k}$.

- Step2: Calculate the acceptance rate $\alpha\left(\theta^{\prime}, \theta_{k}\right)=\min \left\{1, f\left(\theta^{\prime} \mid Y\right) / f\left(\theta_{k} \mid Y\right)\right\}$.

- Step3: Update $\theta_{k}$ as $\theta_{k+1}=\theta^{\prime}$ with probability $\alpha\left(\theta^{\prime}, \theta_{k}\right)$ but otherwise set $\theta_{k+1}=\theta_{k}$ and re-sample from 1.

While running this $\mathrm{M}-\mathrm{H}$ algorithm, we can find, by given any initial guess $\theta_{0}$, the samples will come to a stable Markov chain after a burn-in time $k^{*}$. In other word, unlike common Newton-type iterative regularization methods (for example, the Levenberg-Marquardt algorithm), the MCMC algorithm does not highly depend on the initial guess and the mean value

$$
\theta_{\mathbf{C M}} \approx \frac{1}{K-k^{*}} \sum_{k=k^{*}+1}^{K} \theta_{k}
$$

always reaches the global minimum after a sufficiently long sampling time.

\section{Numerical examples}

In this section, we generate numerically an exact artificial data set $F(\theta)$ and let (10) be the numerical data. In the rest of this paper, we assume the trend $\mu(y)$ has the form:

$$
\mu(y)=r+\alpha y+\beta y^{2}+\gamma y^{3},
$$

where $\alpha, \beta, \gamma$ are the unknown constant. We also assume the measurement data $Y$ has the form:

$$
Y=F(\theta)+\varepsilon,
$$

where random error $\varepsilon$ contains both the random measurement error and the numerical error. By reconstructing the parameters by the $\mathrm{M}-\mathrm{H}$ method, we simultaneously estimate $\alpha, \beta, \gamma$ and $\sigma_{0}$ from the measured data $Y$ in (15).

\subsection{Direct problems}

In this section, we assume $r=0$ and solve the direct problem for (9) by the numerical Crank-Nicholson scheme:

$$
\begin{aligned}
a_{j} U_{i+1, j+1}+(1+b) & U_{i+1, j}+c_{j} U_{i+1, j-1} \\
& =-a_{j} U_{i, j+1}+(1-b) U_{i, j}-c_{j} U_{i, j-1}
\end{aligned}
$$

where $U_{i, j}=U\left(t_{i}, y_{j}\right)$, and

$$
\begin{gathered}
a_{j}=-\frac{\Delta \tau}{4(\Delta y)^{2}}\left\{\sigma_{0}^{2}+\Delta y\left(\frac{1}{2} \sigma_{0}^{2}-\left(\alpha y+\beta y^{2}+\gamma y^{3}\right)\right)\right\} \\
b=\frac{\Delta \tau}{2(\Delta y)^{2}} \\
c_{j}=-\frac{\Delta \tau}{4(\Delta y)^{2}}\left\{\sigma_{0}^{2}-\Delta y\left(\frac{1}{2} \sigma_{0}^{2}-\left(\alpha y+\beta y^{2}+\gamma y^{3}\right)\right)\right\} .
\end{gathered}
$$


82 Here, we took a uniform grid

$$
\begin{aligned}
\tilde{\omega}=\left\{\left(\tau_{i}, y_{j}\right): \tau_{i} \in\left(0, \tau^{*}\right), y_{j}\right. & \in I_{1.5}=(-1.5,1.5), \\
i & =1,2, \cdots, 400, j=1,2, \cdots, 100\}
\end{aligned}
$$

83 with artificial zero Dirichlet boundary conditions at $y=-1.5$ and 1.5 , such as $U_{i, 1}=1$ and $U_{i, 100}=0$, 84 and $\Delta \tau=\tau_{i+1}-\tau_{i}=0.001, \Delta y=y_{j+1}-y_{j}=\frac{1}{33}$.

Then (9) can be given in the matrix form:

$$
\mathbf{u}_{i+1}=\mathbf{A}^{-1} \mathbf{B u}_{i}-2 c_{2} \mathbf{A}^{-1} \mathbf{e}_{98}
$$

where $\mathbf{u}_{\mathbf{i}}=\left(U_{i, 2}, U_{i, 3}, \cdots, U_{i, 99}\right)^{T}, \mathbf{e}_{98}=(1,0, \cdots, 0)^{T}$ and

$$
\mathbf{A}=\left(\begin{array}{cccccc}
1+b & a_{2} & 0 & 0 & \ldots & 0 \\
c_{3} & 1+b & a_{3} & 0 & \ldots & 0 \\
0 & c_{4} & 1+b & a_{4} & \ldots & 0 \\
\vdots & & \ddots & \ddots & \ddots & \vdots \\
0 & & & c_{98} & 1+b & a_{98} \\
0 & \ldots & & 0 & c_{99} & 1+b
\end{array}\right),
$$

$$
\mathbf{B}=\left(\begin{array}{cccccc}
1-b & -a_{2} & 0 & 0 & \ldots & 0 \\
-c_{3} & 1-b & -a_{3} & 0 & \ldots & 0 \\
0 & c_{4} & 1-b & -a_{4} & \ldots & 0 \\
\vdots & & \ddots & \ddots & \ddots & \vdots \\
0 & & & -c_{98} & 1-b & -a_{98} \\
0 & \ldots & & 0 & -c_{99} & 1-b
\end{array}\right)
$$

86 4.2. Inverse problem solution by MCMC

Table 1 shows the true values and parameter settings in $\mathrm{M}-\mathrm{H}$ Algorithm.

Table 1. Parameter setting in $\mathrm{M}-\mathrm{H}$ Algorithm.

\begin{tabular}{c||c|c|c|c}
\hline \hline Parameters & $\alpha$ & $\beta$ & $\gamma$ & $\sigma_{0}$ \\
\hline True value & 1 & 1 & 1 & 1 \\
\hline$\sigma_{\theta}$ & 0.01 & 0.01 & 0.01 & 0.01 \\
\hline \hline
\end{tabular}

In the following examples, the relative noise in all the observations $Y$ is assumed as $1 \%$ and $5 \%$, and the prior distribution $f(\theta)$ of unknowns is $\left(\alpha, \beta, \gamma, \sigma_{0}\right)=1$. That is, we can say

$$
f_{\text {prior }}(\theta)=\mathbf{1}_{\left[\alpha^{\min }, \alpha^{\max }\right]}(\alpha) \cdot \mathbf{1}_{\left[\beta^{\min }, \beta^{\max }\right]}(\beta) \cdot \mathbf{1}_{\left[\gamma^{\min }, \gamma^{\max ]}\right]}(\gamma) \cdot \mathbf{1}_{\left[\sigma_{0}^{\min }, \sigma_{0}^{\max }\right]}\left(\sigma_{0}\right)
$$

and the intervals $\left[\alpha^{\min }, \alpha^{\max }\right],\left[\beta^{\min }, \beta^{\max }\right],\left[\gamma^{\min }, \gamma^{\max }\right]$ and $\left[\sigma_{0}^{\min }, \sigma_{0}^{\max }\right]$ are large enough so that all $\left(\alpha, \beta, \gamma, \sigma_{0}\right)^{\prime}$ s appearing in the Markov chain fall into these intervals. Here, we set the the indicator function as

$$
\mathbf{1}_{A}(a)= \begin{cases}1 & a \in A \\ 0 & a \notin A\end{cases}
$$


88

89

90

General uniform distributions can be used for $f(\theta)$ if we use the prior-reversible proposal that satisfies $f(\theta) q\left(\theta^{\prime} \mid \theta\right)=f\left(\theta^{\prime}\right) q\left(\theta \mid \theta^{\prime}\right)$ (see for example [13]). On the other hand, if we choose $f(\theta)$ as a Gaussian distribution, this will turn out to be the Tikhonov regularization term in the cost function.

For comparison, we particularly consider the Levenberg-Marquardt algorithm $[18,20]$. That is, the recovery of $\theta=\left(\alpha, \beta, \gamma, \sigma_{0}\right)^{T}$ is computed by the iteration given by

$$
\theta_{k+1}=\theta_{k}+\left[F^{\prime}\left(\theta_{k}\right)^{T} F^{\prime}\left(\theta_{k}\right)+\lambda I\right]^{-1} F^{\prime}\left(\theta_{k}\right)^{T}\left(U-F\left(\theta_{k}\right)\right),
$$

where $F^{\prime}(a)$ is the Jacobian matrix and the parameter $\lambda$ is nonnegative. This algorithm can be implemented for example by an inner embedded program lsqcurvefit in MATLAB 2018a.

Example 1: In this example, we set the initial guess of $\left(\alpha, \beta, \gamma, \sigma_{0}\right)$ as $(0,0,0,0)$. Figure 1, Figure3, Figure 5, Figure7 are the trace plots of the chain for $\left(\alpha, \beta, \gamma, \sigma_{0}\right)$, respectively. We can see that the chain mixes well. Moreover recovered results for the posterior probability density function are presented in Figure 2, Figure4, Figure 6, Figure8, and Table 2. From these results the recovery of $\left(\alpha, \beta, \gamma, \sigma_{0}\right)$ represents an excellent approximation of the ture value $(1,1,1,1)$. Here, "Mean value(with $1 \%$ noise) and Mean value(with 5\% noise)" in Table 2 are the average of the value of the iteration time 30000 after burn-in time 5000. For comparison, the converged recovery of $\left(\alpha, \beta, \gamma, \sigma_{0}\right)$ obtained by the Levenberg-Marquardt algorithm for the measured data with 5\% noise is also provided in Table 2.

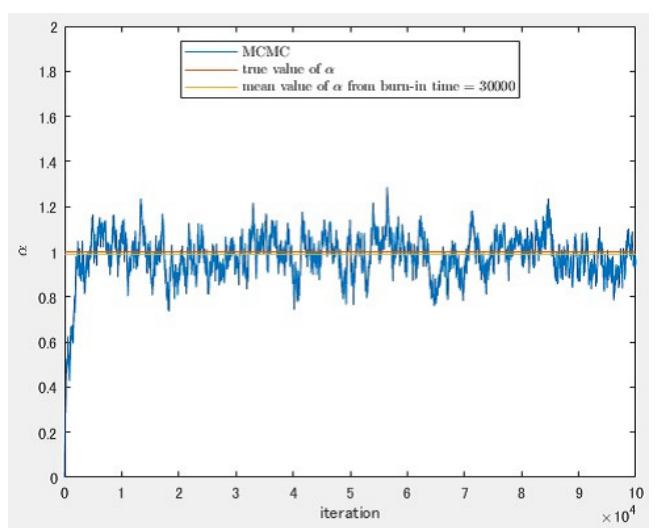

Figure 1. The trace plot of $\alpha$

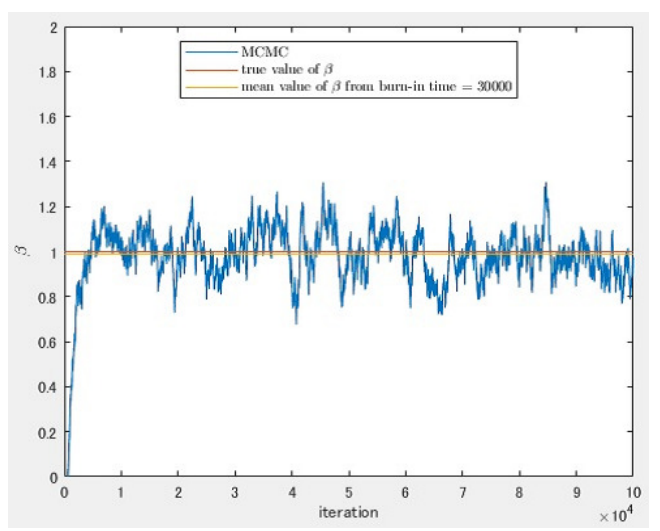

Figure 3. The trace plot of $\beta$

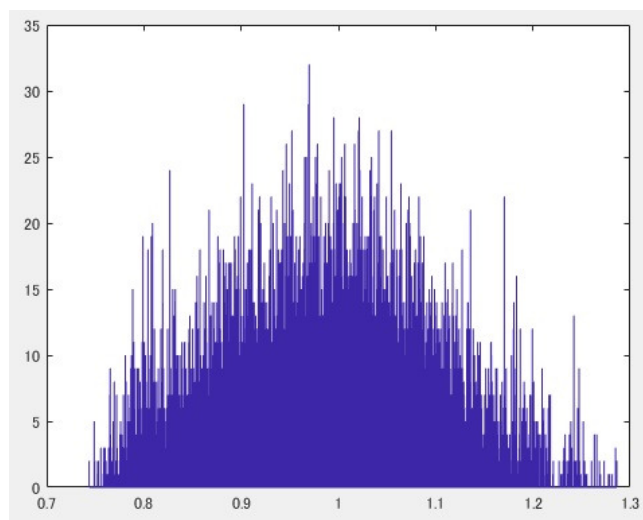

Figure 2. The posterior density for $\alpha$ with $1 \%$ noise added into the data

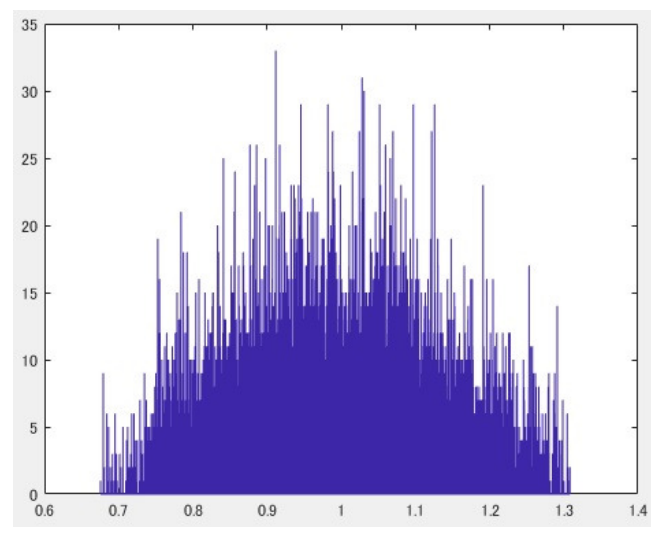

Figure 4. The posterior density for $\beta$ with $1 \%$ noise added into the data 


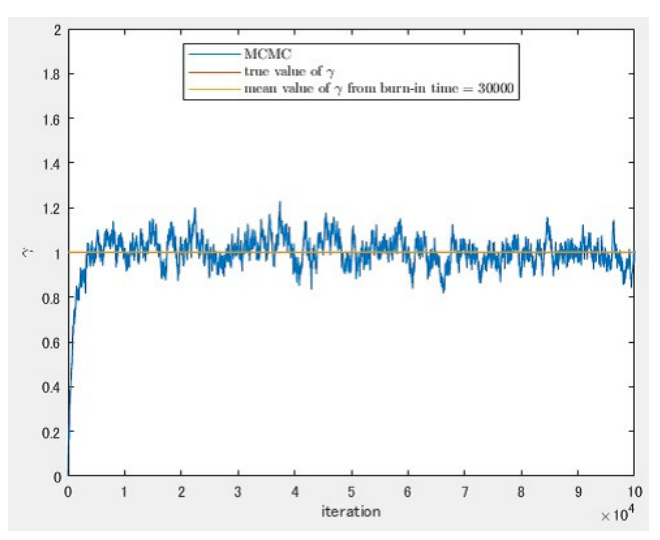

Figure 5. The trace plot of $\gamma$

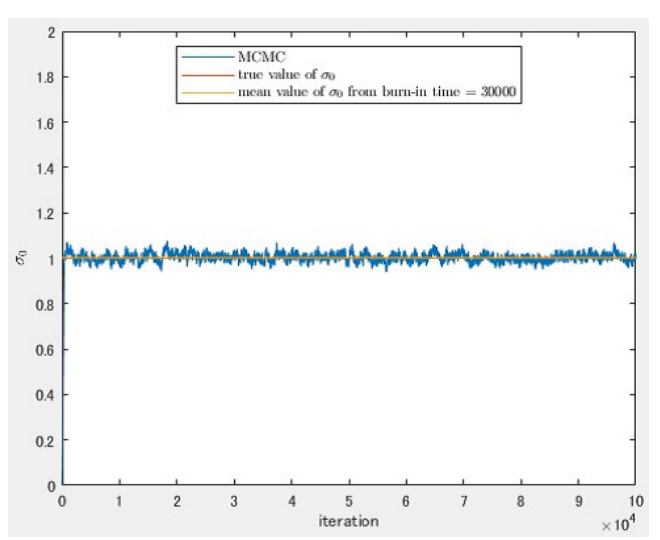

Figure 7. The trace plot of $\sigma_{0}$

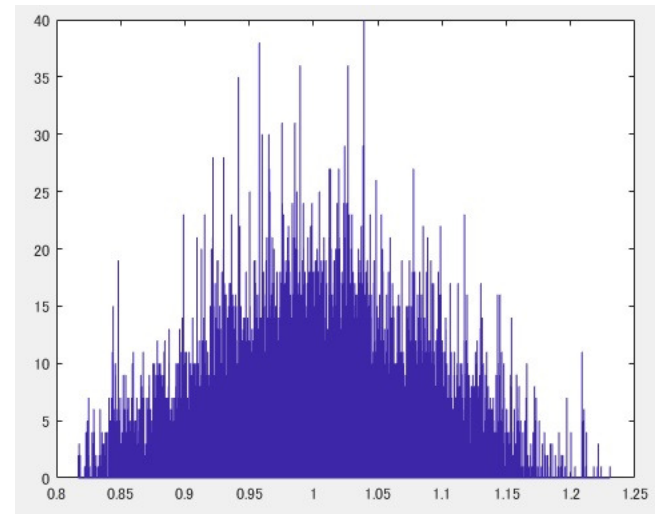

Figure 6. The posterior density for $\gamma$ with $1 \%$ noise added into the data

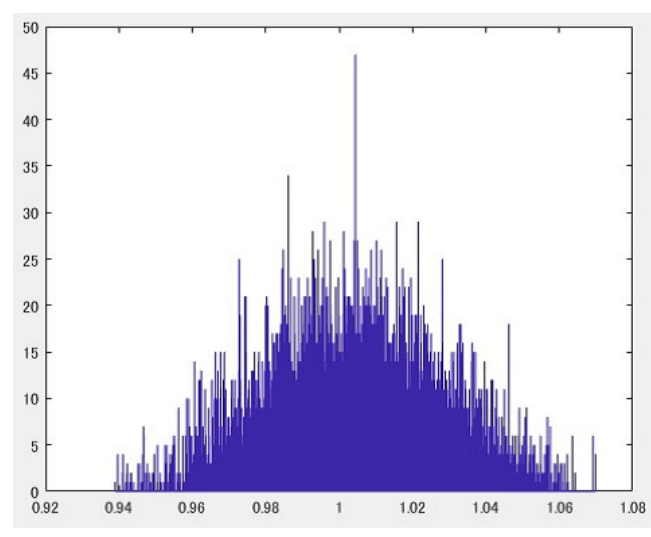

Figure 8. The posterior density for $\sigma_{0}$ with $1 \%$ noise added into the data 
Table 2. Recovery results of $\left(\alpha, \beta, \gamma, \sigma_{0}\right)$.

\begin{tabular}{c||c|c|c|c}
\hline \hline Parameters & $\alpha$ & $\beta$ & $\gamma$ & $\sigma_{0}$ \\
\hline \hline Initial guess & 0 & 0 & 0 & 0 \\
\hline Mean value(with 1\%noise) & 0.9887 & 0.9888 & 1.0022 & 1.0030 \\
\hline Result of LM & 0.9895 & 0.9936 & 1.0054 & 1.0031 \\
\hline Mean value(with 5\% noise) & 1.0556 & 0.9881 & 0.9473 & 0.9912 \\
\hline Result of LM & 1.0662 & 0.9991 & 0.9504 & 0.9894 \\
\hline True value & 1 & 1 & 1 & 1 \\
\hline \hline
\end{tabular}

\section{Example 2:}

In this example, the initial guess of $\left(\alpha, \beta, \gamma, \sigma_{0}\right)$ was set $(3.5,3.5,3.5,3.5)$ to the value far from the true value $(1,1,1,1)$. The evolutions of the MCMC sampled $\alpha, \beta, \gamma$ and $\sigma_{0}$ are shown in Figure 9, Figure11, Figure 13, Figure15 respectively, and we can see that the chain mixes well. Moreover recovered results for the posterior probability density function are presented in Figure 10, Figure12, Figure 14, Figure16, and Table 3. From these results the recovery of $\left(\alpha, \beta, \gamma, \sigma_{0}\right)$ represents an excellent approximation of the ture value $(1,1,1,1)$. The divergent recovery of $\left(\alpha, \beta, \gamma, \sigma_{0}\right)$ obtained by the Levenberg-Marquardt algorithm for the measured data with $5 \%$ noise is also shown in Table 3.

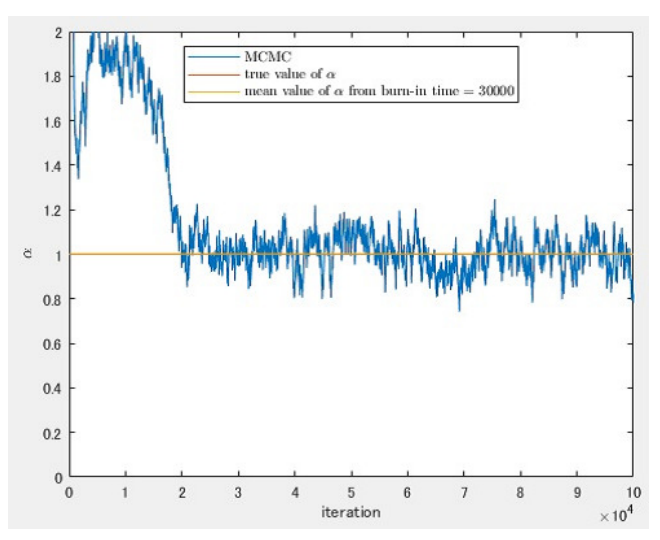

Figure 9. The trace plot of $\alpha$

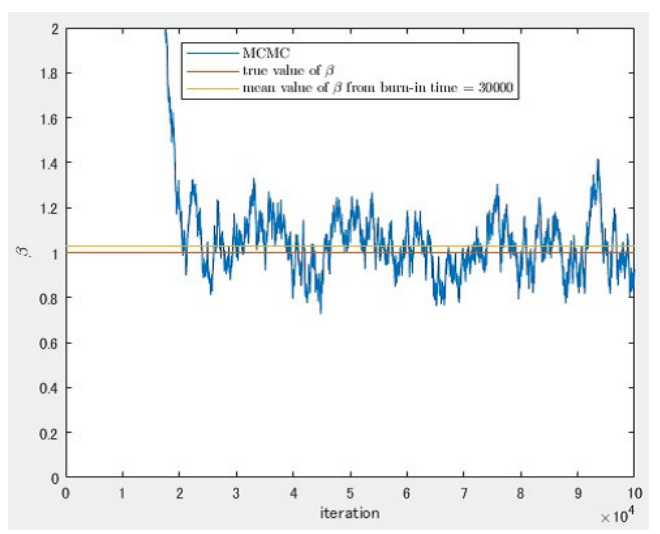

Figure 11. The trace plot of $\beta$

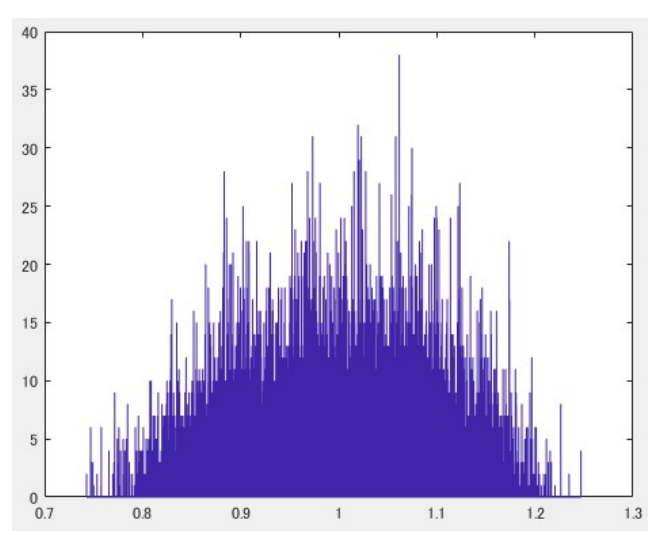

Figure 10. The posterior density for $\alpha$ with $1 \%$ noise added into the data

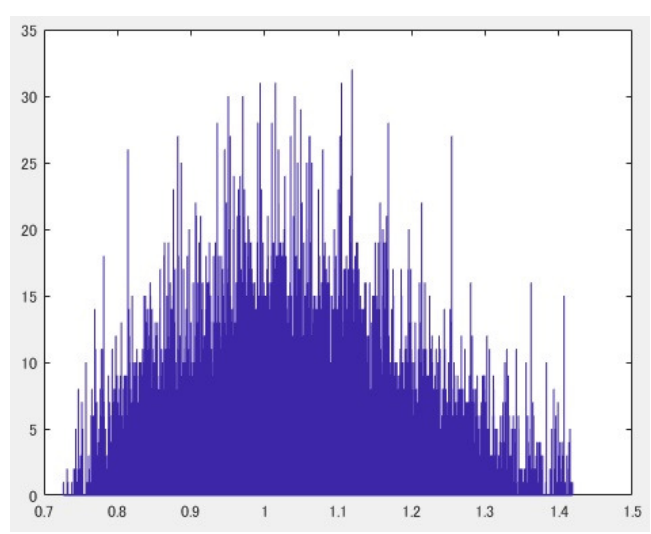

Figure 12. The posterior density for $\beta$ with $1 \%$ noise added into the data 


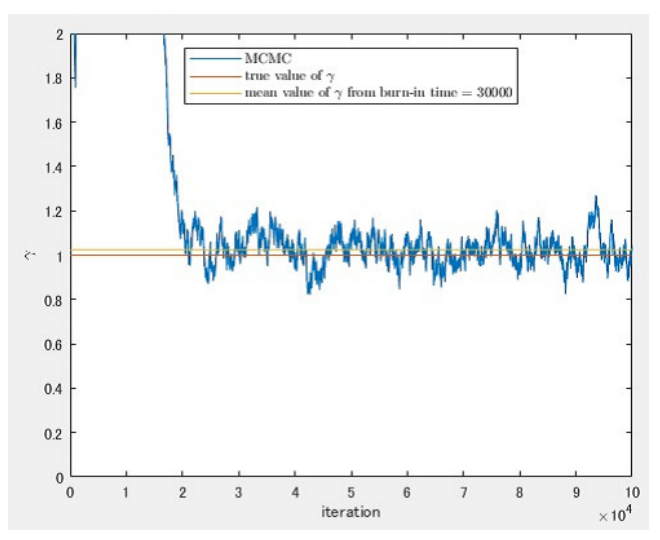

Figure 13. The trace plot of $\gamma$

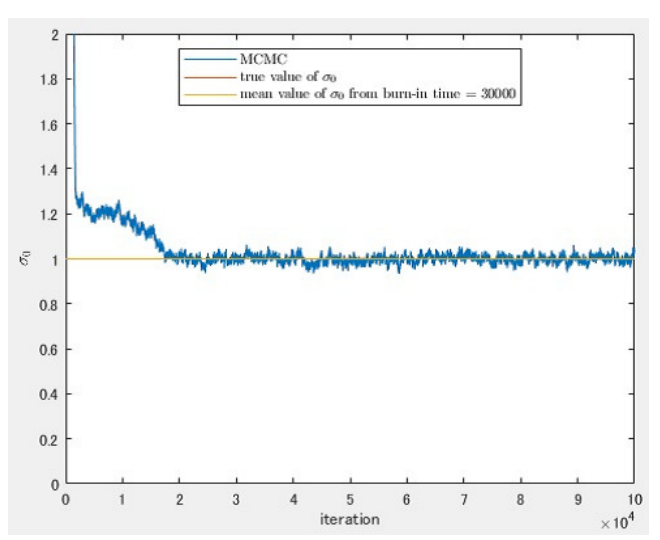

Figure 15. The trace plot of $\sigma_{0}$

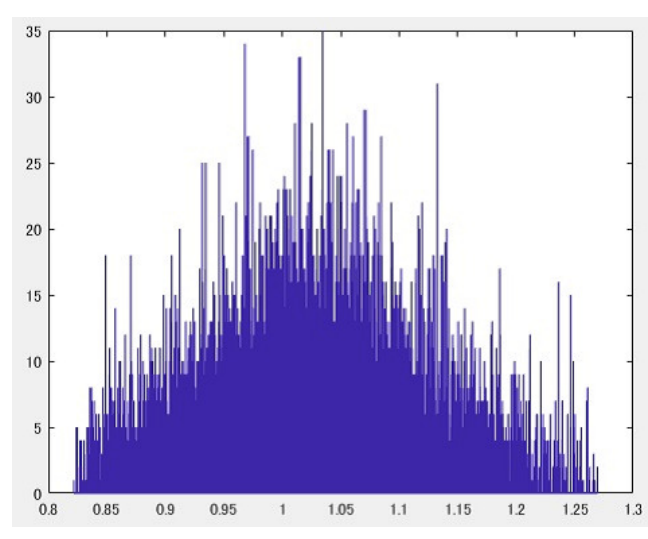

Figure 14. The posterior density for $\gamma$ with $1 \%$ noise added into the data

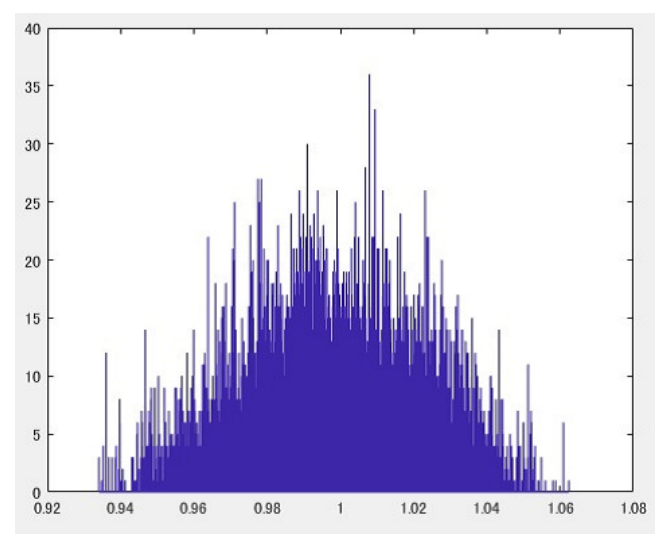

Figure 16. The posterior density for $\sigma_{0}$ with $1 \%$ noise added into the data 
Table 3. Recovery results of $\left(\alpha, \beta, \gamma, \sigma_{0}\right)$.

\begin{tabular}{c||c|c|c|c}
\hline \hline Parameters & $\alpha$ & $\beta$ & $\gamma$ & $\sigma_{0}$ \\
\hline \hline Initial guess & 3.5 & 3.5 & 3.5 & 3.5 \\
\hline Mean value(with 1\% noise) & 1.0022 & 1.0289 & 1.0247 & 0.9992 \\
\hline Result of LM & 0.0001 & 17.9823 & 13.9492 & 2.3801 \\
\hline Mean value(with 5\% noise) & 0.9668 & 0.9830 & 0.9858 & 1.0202 \\
\hline Result of LM & 0.0001 & 16.2754 & 12.6516 & 2.3369 \\
\hline True value & 1 & 1 & 1 & 1 \\
\hline \hline
\end{tabular}

In the case of the initial guess $(0,0,0,0)$, from the results of the MCMC samples in Figure 1 , Figure3, Figure 5, Figure7 and the posterior condition mean values are presented in Table 2, we can see that we succeeded in recovering parameters. And, in the case of the initial guess $(3.5,3.5,3.5,3.5)$ likewise, from the results of the MCMC samples in Figure 9, Figure11, Figure 13, Figure15 and the posterior condition mean values are presented in Table 3, we can see that we succeeded in recovering parameters.

On the other hand, in the case of the initial guess $(0,0,0,0)$, the recoveries obtained by the Levenberg-Marquardt algorithm in Table 2 succeeded as the case of MCMC algorithm. However, in the case of the initial guess $(3.5,3.5,3.5,3.5)$, we could not obtain the results of the recovering parameters by the Levenberg-Marquardt algorithm in Table 3. From these results we observe that parameters are more sensitive to initial values than MCMC algorithm and hence it is less easily recovered.

\section{Conclusions}

In this study, we have established the method of simultaneous estimation of the unknown drift and volatility coefficients from the measured data, by using a Bayesian inference approach(MCMC-MH) based on a partial differential equation of parabolic type. In particular, we took into account an application to real financial markets and dealt with the case with Heaviside function as the initial condition, so-called binary option. In the instantaneous estimation of trend and volatility coefficients, we assumed that the volatility coefficient is a constant and the trend coefficient is a cubic function with three unknown parameters. The posterior distributions of the unknown trend and volatility coefficients were recovered from the measured data by modeling the measurement errors as Gaussian random variables. The posterior state space was explored by the MCMC-M-H method. As confirmed in the numerical results, the Bayesian inference approach(the MCMC algorithm) simultaneously estimated the unknown trend and volatility coefficients from the measured data than the Levenberg-Marquardt algorithm.

There are still several problems we have to settle. First, from the form of our model it is expected that we will be able to apply the results of this study to problems of term structure models for an interest rate. Moreover we will try to identify parameters of another financial model, for instance, such as the model including the dividend yield. Next, we will develop mathematical results (for instance, the uniqueness, stability, and existence) of IOP and extend our approach to two-dimensional cases. Finally, we have to study how to apply our results to the real financial market, and repeat tests.

Author Contributions: Investigation, Ota Y. ; Methodology, Ota Y. and Jiang Y. ; Software , Ota Y. (MCMC) and Jiang Y. (MCMC, LM) ; Validation and Writing-original draft, Ota Y.

Funding: The first author would like to acknowledge the supports from JSPS Grant-in-Aid for Scientific Research (C) 18K03439. The second author was supported by National Natural Science Foundation of China (No. 11771270).

Acknowledgments: In this section you can acknowledge any support given which is not covered by the author contribution or funding sections. This may include administrative and technical support, or donations in kind (e.g., materials used for experiments).

Conflicts of Interest: The authors declare no conflict of interest 


\section{References}

1. Alifanov M. O. 1997 Inverse Heat Transfer Problems, International Series in Heat and Mass Transfer, Springer Verlag.

2. Beck V. J., Blackwell B. and Clair Jr. C. R. 1985 Inverse Heat Conduction: Ill-Posed Problems, Wiley-Interscience.

3. Black F. and Scholes M. 1973 The pricing of options and corporate liabilities, Journal of Political Economy, 81, 637-659.

4. Bouchouev I. and Isakov V. 1999 Uniqueness, stability and numerical methods for the inverse problem that arises in financial markets, Inverse Problems, 15, R95-R116.

5. Bouchouev I., Isakov V. and Valdivia N. 2002 Recovery of volatility coefficient by linearization, Quantitative Finance, Vol2, 257-263.

6. Bunnin F. O., Guo Y. and Ren Y. 2002 Option pricing under model and parameter uncertainty using predictive densities Statistics and Computing, 12(1), 37-44.

7. Cui T., Fox C., and O'fSullivan M.J. 2011 Bayesian calibration of a large-scale geothermal reservoir model by a new adaptive delayed acceptance Metropolis Hastings algorithm, Water Resource Research, 47, W10521.

8. Dupire B. 1994 Pricing with a smil, Risk, 7 18-20.

9. Doi S. and Ota Y. 2018 Application of microlocal analysis to an inverse problem arising from financial markets Inverse Problems. 34, N11.

10. Friedman A. 1983 Partial Differential Equations of Parabolic Type, (Englewood Cliffs, N.J: Prentice-Hall).

11. Haario H., Laine M., Lehtinen M., Saksman E. and Tamminen J. 2004 Markov chain Monte Carlo methods for high dimensional inversion in remote sensing, Journal of the Royal Statistical Society: Series B (Statistical Methodology), 66, 591-608.

12. Hastings, W. 1970 Monte Carlo sampling methods using Markov chains and their application, Biometrika, 57, 97-109.

13. Iglesias M. A., Lin K and Stuart A. M. 2014 Well-posed Bayesian geometric inverse problems arising in subsurface flow, Inverse Problems, 30, 114001.

14. Jacquier E. and Jarrow R. 2000 Bayesian analysis of contingent claim model error, Journal of Econometrics, 94(1-2), 145-180.

15. Jacquier E. and Polson N. 2010 Bayesian methods in finance, Oxford handbook of Bayesian econometrics, 439-512 (Oxford University Press).

16. Kaipio J. and Somersalo E. 2005 Statistical and Computational Inverse Problems. (New York: Springer)

17. Korolev M., Kubo H. and Yagola G. 2012 Parameter identification problem for a parabolic equation-application to the Black-Scholes option pricing model, J. Inverse Ill-posed probl, 20 No.3, 327-337.

18. Levenberg, K. 1944 A method for the solution of certain non-linear problems in least squares, Quarterly Appl. Math., $2,164-168$.

19. Lishang J. and Youshan T. 2001 Identifying the volatility of underlying assets from option prices, Inverse Problems, 17, 137-155.

20. Marquardt D. 1963 An algorithm for least-squares estimation of nonlinear parameters, SIAM J. Appl. Math., 11, 431-441.

21. Martin J., Wilcox L.C., Burstedde C. and Ghattas O. 2012 A stochastic Newton MCMC method for large-scale statistical inverse problems with application to seismic inversion, SIAM Journal on Scientific Computing, 34(3), A1460-A1487.

22. Metropolis N., Rosenbluth A., Rosenbluth M., Teller A. and Teller E. 1953 Equations of state calculations by fast computing machines, J. Chem. Phys., 21(6), 1087-1092.

23. Mitsuhiro M. and Ota Y. 2015 Recovery of Foreign Interest Rates from Exchange Binary Options, Computer Technology and Application, 6, 76-88.

24. Ota Y. and Kaji S. 2016 Reconstruction of local volatility for the binary option model, J. Inverse Ill-posed probl., 24, No.6 727-742.

25. Ota Y., Jiang Y., Nakamura G. and Uesaka M. 2019 Bayesian inference approach to inverse problems in a financial mathematical model, International Journal of Computer Mathematics, submitted.

26. Robert, C. and Casella, G. 2004 Monte Carlo Statistical Methods. (Springer Texts in Statistics)

27. Tunaru R. 2015 Model risk in financial markets: From financial engineering to risk management, World Scientific Pub Co Inc. 
199

200

201

202

203

204

28. Tunaru R. and Zheng T. 2017 Parameter estimation risk in asset pricing and risk management: A Bayesian approach, International Review of Financial Analysis, 53, 80-93.

29. Wang J. and Zabaras N. 2004 A Bayesian inference approach to the inverse heat conduction problem, International Journal of Heat and Mass Transfer, 47, Issues 17-18, 3927-3941.

30. Wang J and Zabaras N. 2005 Hierarchical Bayesian models for inverse problems in heat conduction, Inverse Problems, 21 183-206. 